\title{
Publication trends in human learning and memory: 1962-1982
}

\author{
WILLIAM E. FORRESTER \\ State University of New York, Oswego, New York
}

\begin{abstract}
The relative fates of human learning and memory as research topics in psychology were assessed by an analysis of titles published from 1962 to 1982 . Publications were classified as "learning" or "memory" on the basis of titular descriptions provided by authors of the respective publications. Results indicated that, whereas researchers in 1962 were predominantly concerned with learning, rather than memory, a significant reversal occurred over the ensuing 10 years $(p<.001)$. The subsequent 10 years, however, may be characterized as a period of stability.
\end{abstract}

During its behavioristic period, psychology placed great emphasis on the topic of learning and tended to consider memory as an unimportant concept. Indeed, "memory" virtually disappeared from the lexicon of the discipline. Although the relative fates of learning and memory seem to have changed considerably during the postbehavioristic era, the extent of that change has not been documented in any way. The purpose of this study, therefore, was to provide such documentation by an analysis of publication trends over the 20 -year period beginning in 1962 .

The procedures used to classify publications as falling into the domains of learning or memory were based on the not unreasonable assumption that authors' titular descriptions of their research should reflect the underlying topics of that research. The basic data, therefore, were the frequencies with which specific terms appeared in the titles of joumal articles. Two sources were used to obtain the data. One source was the Journal of Verbal Learning and Verbal Behavior. That journal was introduced in 1962, and, according to Cofer (1968), its dominant theme has been represented by learning and memory. Additional data were obtained from reference lists provided in the four Annual Review chapters devoted to both learning and memory that appeared between 1962 and 1982.

Titles of articles published in Volumes 1 through 20 of the Journal of Verbal Learning and Verbal Behavior $(J V L V B)$ were categorized as "learning," "memory," "both," or "neither." Two separate counts were made: The first used a stringent scoring criterion, and the second, a more lenient criterion. When the stringent scoring criterion was used, articles were placed into the respective categories on the basis of the presence or absence of the specific terms "learning" and "memory." Under the lenient scoring criterion, articles were cate-

Requests for reprints should be sent to the author at the Department of Psychology, State University of New York, Oswego, New York 13126. gorized as "learning" if the term "learning," "acquisition," or "conditioning" appeared in the title. Articles were classified as being concerned with "memory" if the term "memory," "retention," or "recall" appeared.

Data obtained from Annual Review chapters were handled similarly. The specific reviews from which data were obtained were those written by Keppel (1968), Postman (1975), Tulving and Madigan (1970), and Wickelgren (1981).

The total number of articles published in each volume of $J V L V B$ was quite variable over the 20 -year period. The general trend was one of increasing numbers of articles over the first 7 years of the journal's existence (from a total of 70 in Volume 1 to 212 in Volume 7) and decreasing numbers through the latter part of the period. The number of articles published in Volume 20, for example, was 48 . Because of this variability, the data were converted from frequencies to proportions.

Although the pattern of results revealed obvious changes in the relative numbers of articles in the learning

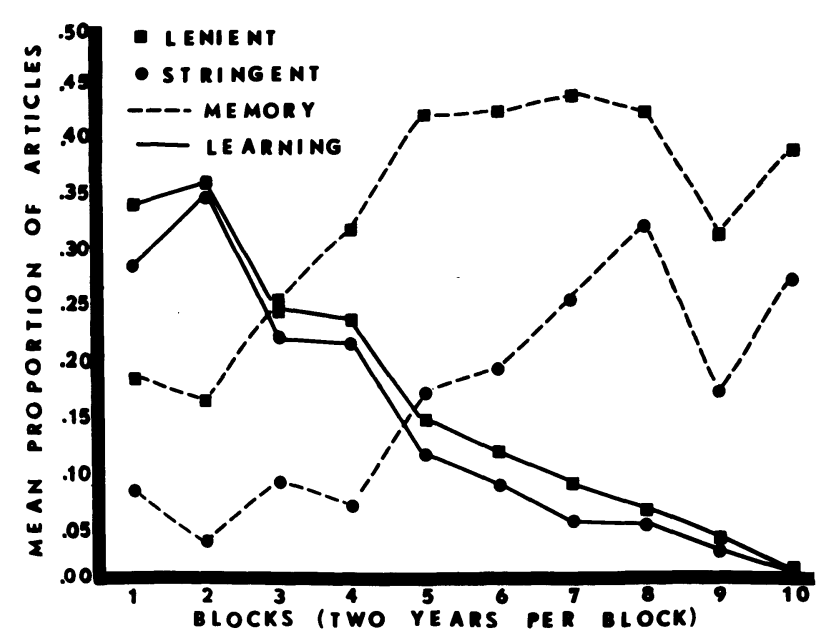

Figure 1. Mean annual proportions of learning and memory articles published in $J V L V B$. 
Table 1

Proportion of References to Learning and Memory in Four Annual Review Chapters

\begin{tabular}{|c|c|c|c|c|c|}
\hline \multirow[b]{2}{*}{ Authors } & \multirow[b]{2}{*}{$\mathrm{n}$} & \multicolumn{2}{|c|}{ Stringent Scoring } & \multicolumn{2}{|c|}{ Lenient Scoring } \\
\hline & & Learning & -Memory & Learning & Memory \\
\hline Keppel (1968) & 253 & .35 & .10 & .37 & .22 \\
\hline Tulving \& Madigan (1970) & 189 & .17 & .31 & .18 & .49 \\
\hline Postman (1975) & 214 & .09 & .34 & .10 & .63 \\
\hline Wickelgren (1981) & 122 & .06 & .29 & .07 & .34 \\
\hline
\end{tabular}

Note $-n=$ total number of references cited.

and memory categories, the proportion of total articles for these two categories combined was relatively stable throughout the 20-year period. Results obtained with the stringent scoring method showed that approximately $30 \%$ of the articles published each year were concerned with learning or memory (mean proportion $=.31$, $\mathrm{SD}=.07)$. Under the lenient method of scoring, half of $J V L V B$ 's articles each year fell into these two categories $($ mean proportion $=.50, \mathrm{SD}=.08)$.

With regard to differences between the learning and the memory classifications, the pattern of results (shown in Figure 1) reflects a downward trend for the former and a corresponding increase for the latter. The major difference attributable to the lenient-stringent distinction in scoring was the earlier crossover point in the respective publication trends when lenient scoring was used. In short, the terms "recall" and "retention" began to appear in titles before "memory" per se did.

Because the question of interest in this study concerned the relative fates of learning and memory over the period surveyed, only articles falling into these two respective categories were subjected to statistical analysis. Prior to being analyzed, the data for the 20-year period were grouped into 10 blocks of 2 years each. Initial analyses were performed to determine whether the relative numbers of articles classified as learning or memory articles had changed to a significant degree over the blocks of time. If so, further analyses were performed to find the locus of the effect. The procedures formulated by Brandt and Snedecor (cited in Cochran, 1954) were used throughout.

\section{Table 2}

Partitioning of Chi-Squares for Annual Review References

\begin{tabular}{lrcc}
\hline \multicolumn{1}{c}{ Source } & SS & df & $\chi^{2}$ \\
\hline & \multicolumn{4}{c}{ Stringent } \\
Keppel (K) vs. Tulving (T) & 8.85 & 1 & $35.95^{*}$ \\
Postman (P) vs. Wickelgren (W) & .07 & 1 & .28 \\
K \& T vs. P \& W & 12.70 & 1 & $51.59^{*}$ \\
Overall & 21.62 & 3 & $87.82^{*}$ \\
& \multicolumn{4}{c}{ Lenient Scoring } \\
Keppel (K) vs. Tulving (T) & 8.71 & 1 & $41.33^{*}$ \\
Postman (P) vs. Wickelgren (W) & .08 & 1 & .38 \\
K \& T vs. P \& W & 15.41 & 1 & $73.11^{*}$ \\
Overall & 24.20 & 3 & $114.82^{*}$ \\
\hline
\end{tabular}

*Indicates $p<.001$.
Analysis of the data obtained with stringent scoring showed that the changes that occurred over the 20 years, with respect to learning and memory articles, were highly significant $\left[\chi^{2}(9)=178.70, N=542, S S=44.38\right.$, $\mathrm{p}<.001]$. Partitioning of the overall chi-square into additive components revealed that most of the variability could be attributed to changes that occurred within Volumes $1-10\left[\chi^{2}(4)=31.45, \mathrm{~N}=293, \mathrm{SS}=\right.$ $7.81, \mathrm{p}<.001]$ and between Volumes 1-10 and Volumes $11-20\left[\chi^{2}(1)=140.49, \mathrm{SS}=34.89, \mathrm{p}<.001\right]$. No significant changes were found within Volumes 11$20\left[\chi^{2}(4)=6.76, N=249, \mathrm{SS}=1.68\right]$. The same pattern of results was found when the data obtained with lenient scoring were analyzed. The overall chi-square was again highly significant $\left[\chi^{2}(9)=140.48, N=895\right.$, $\mathrm{SS}=32.86, \mathrm{p}<.001]$, as were the Volumes $1-10$ versus Volumes $11-20$ component $\left[\chi^{2}(1)=84.35\right.$, SS $=19.73, p<.001]$ and the within-Volumes $1-10$ component $\left[\chi^{2}(4)=49.72, \mathrm{~N}=334, \mathrm{SS}=11.63\right.$, $\mathrm{p}<.001]$. As was the case with stringent scoring, no significant changes were found within Volumes 11-20 $\left[\chi^{2}(4)=6.41, \mathrm{~N}=561, \mathrm{SS}=1.50\right]$

The historical trends indicated by the JVLVB data were equally apparent in the data obtained from Annual Review chapters. The relative numbers of reference articles falling into the categories under consideration are shown in Table 1. Analysis of the data (i.e., learning vs. memory $x$ four chapters) followed the same general procedures as described previously. The results of analyses performed on the data obtained under the two scoring methods are summarized in Table 2 . As was the case for $J V L V B$ data, the pattern of results was essentially unaffected by the method of scoring. In addition, the pattern of specific results for the Annual Review data was quite similar to the $J V L V B$ results. Thus, significant changes in the relative numbers of learning and memory articles were found in the comparison between the early reviews (i.e., Keppel, 1968, and Tulving \& Madigan, 1970) and between those two reviews and the ones by Postman (1975) and Wickelgren (1981). Differences between the latter, however, were nonsignificant.

\section{DISCUSSION}

The results of this survey indicated that, in 1962, research in the areas of human learning and memory was predominantly concerned with the former, rather than the latter, but that a significant reversal occurred over the next 10 years. The subse- 
quent 10 years, however, may be viewed as a period of stability. Although the results of this survey are quite clear, their interpretation is not. The problem arises from the fact that the primary data for the study were the titular descriptions of research provided by the researchers themselves. It may very well be that some studies that were placed in the "learning" category for purposes of this survey might now be viewed as investigations of memory processes. Articles containing the expression "freerecall learning" in their titles would be examples of this. In retrospect, such articles may be seen as being concerned with organizational processes of memory. Whether they were so considered by their authors at the time they were reported is unknown. How they will be viewed 20 years hence is also unknown. Perhaps they will then be treated as investigations of perceptual processes.

\section{REFERENCES}

Cochran, W. G. (1954). Some methods for strengthening the common $\chi^{2}$ tests. Biometrics, 10, 417-451.

Cofer, C. N. (1978). Origins of the Journal of Verbal Learning and Verbal Behavior. Journal of Verbal Learning and Verbal Behavior, 17, 113-126.

KePPEL, G. (1968). Verbal learning and memory. Annual Review of Psychology, 19, 169-202.

Postman, L. (1975). Verbal learning and memory. Annual Review of Psychology, 26, 291-335.

Tulving, E. \& Madigan, S. A. (1970). Memory and verbal learning. Annual Review of Psychology, 21, 437-484.

Wickelgren, W. A. (1981). Human Learning and memory. Annual Review of Psychology, 32, 21-52.

(Manuscript received for publication November 21, 1983.) 